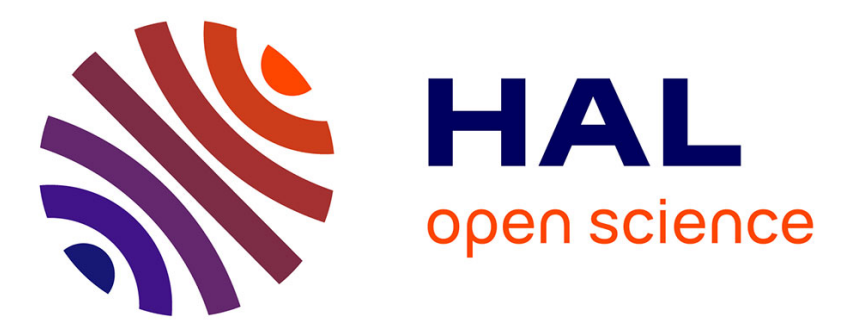

\title{
Noise from scattered light in Virgo's second science run data
}

\author{
T Accadia, F Acernese, F Antonucci, P Astone, G Ballardin, F Barone, M.
} Barsuglia, Th S Bauer, M G Beker, A Belletoile, et al.

\section{> To cite this version:}

T Accadia, F Acernese, F Antonucci, P Astone, G Ballardin, et al.. Noise from scattered light in Virgo's second science run data. 14th Gravitational Wave Data Analysis Workshop (GWDAW-14), Jan 2010, rome, Italy. pp.194011, 10.1088/0264-9381/27/19/194011 . hal-00629992

\section{HAL Id: hal-00629992 https://hal.science/hal-00629992}

Submitted on 7 Oct 2011

HAL is a multi-disciplinary open access archive for the deposit and dissemination of scientific research documents, whether they are published or not. The documents may come from teaching and research institutions in France or abroad, or from public or private research centers.
L'archive ouverte pluridisciplinaire HAL, est destinée au dépôt et à la diffusion de documents scientifiques de niveau recherche, publiés ou non, émanant des établissements d'enseignement et de recherche français ou étrangers, des laboratoires publics ou privés. 


\section{Noise from scattered light in Virgo's second science run data}

T. Accadia ${ }^{11}$, F. Acernese Acc $^{5 a c}$ F. Antonucci ${ }^{8 a}$, P. Astone ${ }^{8 a}$, G. Ballardin ${ }^{2}$, F. Barone ${ }^{5 a c}$, M. Barsuglia ${ }^{1}$, Th.S. Bauer ${ }^{13 a}$, M.G. Beker ${ }^{13 a}$, A. Belletoile ${ }^{11}$, S. Birindelli ${ }^{14 a}$, M. Bitossi ${ }^{7 a}$, M.A. Bizouard ${ }^{10 a}$, M. Blom ${ }^{13 a}$, F. Bondu ${ }^{14 b}$, L. Bonelli $^{7 a b}$,

R. Bonnand ${ }^{12}$, V. Boschi ${ }^{7 a}$, L. Bosi ${ }^{6 a}$, B. Bouhou ${ }^{1}$, S. Braccini ${ }^{7 a}$, C. Bradaschia ${ }^{7 a}$, A. Brillet ${ }^{14 a}$, V. Brisson ${ }^{10 a}$, R. Budzyński ${ }^{16 b}$,

T. Bulik ${ }^{16 c d}$, H.J. Bulten ${ }^{13 a b}$, D. Buskulic ${ }^{11}$, C. Buy ${ }^{1}$, G. Cagnoli ${ }^{3 a}$, E. Calloni ${ }^{5 a b}$, E. Campagna ${ }^{3 a b}$, B. Canuel ${ }^{2}$,

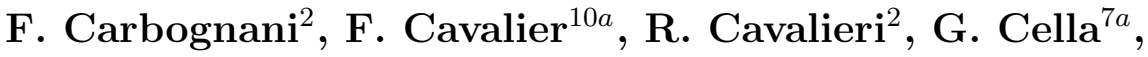

E. Cesarini ${ }^{3 b}$, E. Chassande-Mottin ${ }^{1}$, A. Chincarini ${ }^{4}$, F. Cleva ${ }^{14 a}$, E. Coccia ${ }^{9 a b}$, C.N. Colacino ${ }^{7 a b}$, J. Colas ${ }^{2}$, A. Colla ${ }^{8 a b}$, M. Colombini ${ }^{8 b}$, A. Corsi ${ }^{8 a}$, J.-P. Coulon ${ }^{14 a}$, E. Cuoco $^{2}$, S. D’Antonio ${ }^{9 a}$, V. Dattilo ${ }^{2}$, M. Davier ${ }^{10 a}$, R. Day ${ }^{2}$, R. De Rosa ${ }^{5 a b}$, G. Debreczeni ${ }^{17}$, M. del Prete Prc $^{7 a c}$ L. Di Fiore ${ }^{5 a}$, A. Di Lieto ${ }^{7 a b}$, M. Di Paolo Emilio ${ }^{9 a c}$, A. Di Virgilio ${ }^{7 a}$, A. Dietz ${ }^{11}$, M. Drago ${ }^{15 c d}$, V. Fafone ${ }^{9 a b}$, I. Ferrante ${ }^{7 a b}$, F. Fidecaro ${ }^{7 a b}$, I. Fiori ${ }^{2}$, R. Flaminio ${ }^{12}$, J.-D. Fournier ${ }^{14 a}$, J. Franc ${ }^{12}$, S. Frasca ${ }^{8 a b}$, F. Frasconi ${ }^{7 a}$, A. Freise*, M. Galimberti ${ }^{12}$, L. Gammaitoni ${ }^{6 a b}$, F. Garufi ${ }^{5 a b}$, M. E. Gáspár ${ }^{17}$, G. Gemme ${ }^{4}$, E. Genin ${ }^{2}$, A. Gennai ${ }^{7 a}$, A. Giazotto ${ }^{7 a}$, R. Gouaty ${ }^{11}$, M. Granata ${ }^{1}$, C. Greverie ${ }^{14 a}$, G. M. Guidi ${ }^{3 a b}$, J.-F. Hayau ${ }^{14 b}$, H. Heitmann ${ }^{14}$, P. Hello ${ }^{10 a}$, S. Hild ${ }^{* *}$, D. Huet ${ }^{2}$, P. Jaranowski ${ }^{16 e}$, I. Kowalska ${ }^{16 c}$, A. Królak ${ }^{16 a f}$, N. Leroy ${ }^{10 a}$, N. Letendre ${ }^{11}$, T.G.F. Li ${ }^{13 a}$, M. Lorenzini ${ }^{3 a}$, V. Loriette ${ }^{10 b}$, G. Losurdo ${ }^{3 a}$, E. Majorana ${ }^{8 a}$, I. Maksimovic ${ }^{10 b}$, N. Man ${ }^{14 a}$, M. Mantovani ${ }^{7 a c}$, F. Marchesoni ${ }^{6 a}$, F. Marion ${ }^{11}$, J. Marque ${ }^{2}$, F. Martelli ${ }^{3 a b}$, A. Masserot ${ }^{11}$,

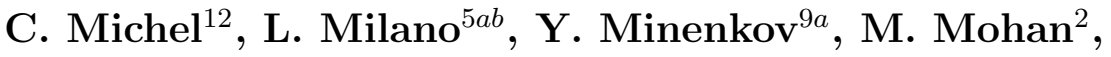
N. Morgado ${ }^{12}$, A. Morgia ${ }^{9 a b}$, S. Mosca ${ }^{5 a b}$, V. Moscatelli ${ }^{8 a}$, B. Mours ${ }^{11}$, I. Neri ${ }^{6 a b}$, F. Nocera ${ }^{2}$, G. Pagliaroli ${ }^{9 a c}$, L. Palladino ${ }^{9 a c}$, C. Palomba ${ }^{8 a}$, F. Paoletti ${ }^{7,2}$, S. Pardi ${ }^{5 a b}$, M. Parisi ${ }^{5 b}$, A. Pasqualetti ${ }^{2}$, R. Passaquieti ${ }^{7 a b}$, D. Passuello ${ }^{7 a}$, G. Persichetti ${ }^{5 a b}$, M. Pichot ${ }^{14 a}$, F. Piergiovanni ${ }^{3 a b}$, M. Pietka ${ }^{16 e}$, L. Pinard ${ }^{12}$, R. Poggiani ${ }^{7 a b}$, M. Prato ${ }^{4}$, G.A. Prodi ${ }^{15 a b}$, M. Punturo ${ }^{6 a}$, P. Puppo ${ }^{8 a}$, D.S. Rabeling ${ }^{13 a b}$, I. Rácz ${ }^{17}$, 
P. Rapagnani ${ }^{8 a b}$, V. Re ${ }^{15 a b}$, T. Regimbau ${ }^{14 a}$, F. Ricci ${ }^{8 a b}$, F. Robinet ${ }^{10 a}$, A. Rocchi ${ }^{9 a}$, L. Rolland ${ }^{11}$, R. Romano ${ }^{5 a c}$, D. Rosińska ${ }^{16 g}$, P. Ruggi ${ }^{2}$, B. Sassolas ${ }^{12}$, D. Sentenac ${ }^{2}$, L. Sperandio ${ }^{9 a b}$, R. Sturani ${ }^{3 a b}$, B.L. Swinkels ${ }^{2}$, A. Toncelli ${ }^{7 a b}$,

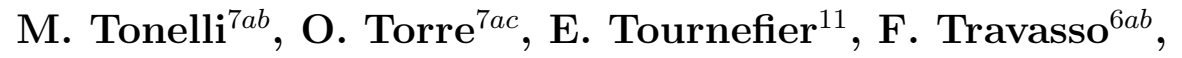
G. Vajente ${ }^{7 a b}$, J.F.J. van den Brand ${ }^{13 a b}$, S. van der Putten ${ }^{13 a}$, M. Vasuth ${ }^{17}$, M. Vavoulidis ${ }^{10 a}$, G. Vedovato ${ }^{15 c}$, D. Verkindt ${ }^{11}$,

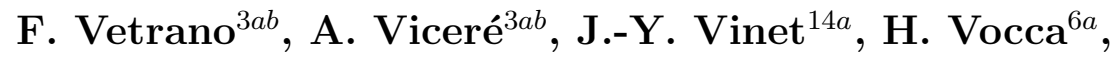
M. Was ${ }^{10 a}$, M. Yvert ${ }^{11}$

${ }^{1}$ AstroParticule et Cosmologie (APC), CNRS: UMR7164-IN2P3-Observatoire de Paris-Université Denis Diderot-Paris 7 - CEA : DSM/IRFU, France

${ }^{2}$ European Gravitational Observatory (EGO), I-56021 Cascina (PI), Italy

${ }^{3}$ INFN, Sezione di Firenze, I-50019 Sesto Fiorentino ${ }^{a}$; Università degli Studi di Urbino 'Carlo Bo', I-61029 Urbino ${ }^{b}$, Italy

${ }^{4}$ INFN, Sezione di Genova; I-16146 Genova, Italy

${ }^{5}$ INFN, Sezione di Napoli ${ }^{a}$; Università di Napoli 'Federico II ${ }^{b}$ Complesso

Universitario di Monte S.Angelo, I-80126 Napoli; Università di Salerno, Fisciano, I-84084 Salerno ${ }^{c}$, Italy

${ }^{6}$ INFN, Sezione di Perugia ${ }^{a}$; Università di Perugia ${ }^{b}$, I-06123 Perugia,Italy

${ }^{7}$ INFN, Sezione di Pisa ${ }^{a}$; Università di Pisa ${ }^{b}$; I-56127 Pisa; Università di Siena, I-53100 Siena ${ }^{c}$, Italy

${ }^{8}$ INFN, Sezione di Roma ${ }^{a}$; Università 'La Sapienza ${ }^{\prime b}$, I-00185 Roma, Italy

${ }^{9}$ INFN, Sezione di Roma Tor Vergata ${ }^{a}$; Università di Roma Tor Vergata, I-00133

Roma $^{b}$; Università dell'Aquila, I-67100 L'Aquila ${ }^{c}$, Italy

${ }^{10}$ LAL, Université Paris-Sud, IN2P3/CNRS, F-91898 Orsay ${ }^{a}$; ESPCI, CNRS, F-75005 Paris ${ }^{b}$, France

${ }^{11}$ Laboratoire d'Annecy-le-Vieux de Physique des Particules (LAPP), IN2P3/CNRS, Université de Savoie, F-74941 Annecy-le-Vieux, France

${ }^{12}$ Laboratoire des Matériaux Avancés (LMA), IN2P3/CNRS, F-69622 Villeurbanne, Lyon, France

${ }^{13}$ Nikhef, National Institute for Subatomic Physics, P.O. Box 41882, 1009 DB

Amsterdam $^{a}$; VU University Amsterdam, De Boelelaan 1081, 1081 HV Amsterdam ${ }^{b}$, The Netherlands

${ }^{14}$ Université Nice-Sophia-Antipolis, CNRS, Observatoire de la Côte d'Azur, F-06304

Nice $^{a}$; Institut de Physique de Rennes, CNRS, Université de Rennes 1, 35042

Rennes $^{b}$, France

${ }^{15}$ INFN, Gruppo Collegato di Trento ${ }^{a}$ and Università di Trento ${ }^{b}$, I-38050 Povo, Trento, Italy; INFN, Sezione di Padova ${ }^{c}$ and Università di Padova ${ }^{d}$, I-35131 Padova, Italy

${ }^{16}$ IM-PAN 00-956 Warsaw $^{a}$; Warsaw University 00-681 Warsaw $^{b}$; Astronomical Observatory Warsaw University 00-478 Warsaw $^{c}$; CAMK-PAN 00-716 Warsaw ${ }^{d}$;

Białystok University 15-424 Bial ystok ${ }^{e}$; IPJ 05-400 Świerk-Otwock ${ }^{f}$; Institute of Astronomy 65-265 Zielona Góra ${ }^{g}$, Poland

${ }^{17}$ RMKI, H-1121 Budapest, Konkoly Thege Miklós út 29-33, Hungary

${ }^{*}$ University of Birmingham, Birmingham, B15 2TT, United Kingdom

** University of Glasgow, Glasgow, G12 8QQ, United Kingdom

E-mail: irene.fiori@ego-gw.it 
Abstract. Virgo is one of the large, ground-based interferometers aimed at detecting
gravitational waves. One of the technical problems limiting its sensitivity is caused
by light in the output beams which is back-scattered by seismically excited surfaces
and couples back into the main beam of the interferometer. The resulting noise was
thoroughly studied, measured and mitigated before Virgo's second science run (VSR2).
The residual noise during VSR2, which increases in periods with large microseism
activity, is accurately predicted by the theoretical model. The scattered light has been
associated with transient events in the gravitational-wave signal of the interferometer.

\section{Introduction}

The Virgo detector, which is aimed at detecting gravitational waves, is a power-recycled Michelson interferometer with $3 \mathrm{~km}$ long Fabry-Perot cavities in its arms. It features an ultra-stable Nd-YAG laser that sends $25 \mathrm{~W}$ into the interferometer and test-mass mirrors that are seismically isolated from ground by means of a very efficient multistage suspension system (Super-Attenuators). All the core optics are located in an ultra-high vacuum system. Virgo measures signals in a frequency-band between 10 and $4000 \mathrm{~Hz}$ and reaches a strain sensitivity as low as $1 \cdot 10^{-22} / \sqrt{ } \mathrm{Hz}$.

Virgo has recently concluded its second science run (VSR2) in coincidence with the LIGO and GEO detectors. The sensitivity reached during VSR2 was close to its design goal. Noticeably, all major fundamental and technical noise-sources have been modeled and their incoherent sum closely matches the measured residual noise [1]. One of the sources of technical noise is related to diffused light, which has been studied extensively and has been mitigated for the case of light scattered inside the vacuum system $[2,3,4]$. Similarly, light scattered from optics located on benches outside the vacuum system can introduce noise [5].

At Virgo, in-air benches are placed at the input port (External Injection Bench, EIB), output port (External Detection Bench, EDB) and behind the terminal mirrors (North and West-End Benches, NEB and WEB), which transmit just a tiny fraction of light for controlling the alignment of the interferometer. Optical powers between 100 $\mathrm{mW}$ and a few Watt are received by these benches. Inevitably, a tiny fraction of this light gets diffused by the optical components (lenses, mirrors, photo-detectors, beam dumps) on the benches, which move due to seismic excitation. Part of this light, which is phase-modulated by the motion of the optics, scatters backwards and recombines with the main optical beam resonating inside the interferometer.

Scattered light from external optics was found to be limiting the sensitivity of Virgo after its first science run (VSR1, 2007) [6]. Section 2 describes the model developed for scattered-light noise as well as measurements and mitigations performed on the interferometer. As predicted by the model, some residual noise was still present during VSR2. Section 3 shows how this noise causes transient events in case of bad weather. 


\section{Back-scattered light from in-air benches}

This section will discuss the case of light scattered by Virgo's terminal benches. A more detailed analysis and results for the other benches can be found in reference [8].

\subsection{Model}

It is assumed that all optics move coherently with the bench, which is valid since only bench motion at frequencies below those of optical mount resonances is considered (e.g. $f \leq 100 \mathrm{~Hz}$ ).

Consider the light that is resonating in one of the long Fabry-Perot cavities with amplitude $\overrightarrow{A_{0}}$. A fraction $T$ of the optical power (40 ppm for Virgo) is transmitted by the end-mirror to the terminal bench. Each optical component on this bench diffuses the impinging light according to its bidirectional reflectance distribution function (BRDF). Overall, a tiny fraction $f_{s c}$ of the optical power scatters exactly backwards and recouples to the optical field resonating inside the Fabry-Perot. This is the back-scattered beam with amplitude $\overrightarrow{A_{s c}}$. Its power is suppressed by the mirror transmission factor $(T)$ and then multiplied by the Fabry-Perot optical gain $(2 F / \pi)$, where $F$ is the finesse. The amplitude of the scattered beam is therefore $A_{s c}=A_{0} T \sqrt{\frac{2 F}{\pi}} \sqrt{f_{s c}}$. The scattered field carries a phase noise with respect to the static Fabry-Perot field because its optical path length is modulated by the displacement of the scattering optics. Its phase angle is

$$
\phi_{s c}(t)=\frac{4 \pi}{\lambda}\left(x_{0}+\delta x_{o p t}(t)\right)=\phi_{0}+\delta \phi_{s c}(t),
$$

where $\delta x_{\text {opt }}$ is the displacement of the optics along the direction of beam (essentially the displacement of bench in the horizontal plane), $x_{0}$ is the static optical path and $\lambda$ is the optical wavelength $(1064 \mathrm{~nm})$.

The total field inside the arm cavity is given by $\overrightarrow{A_{t o t}}=\overrightarrow{A_{0}}+\overrightarrow{A_{s c}}$, its phase angle is the phase noise added to the interferometer beam: $\delta \Phi=\frac{A_{s c}}{A_{0}} \cdot \sin \phi_{s c}$. Since the measured gravitational-wave strain is proportional to this phase, the noise introduced by the scattered light $h_{s c}$ can be written as

$$
h_{s c}(t)=\frac{\lambda}{8 F L} \delta \Phi=K \cdot \sqrt{f_{s c}} \cdot \sin \left(\phi_{s c}\right)=G \cdot \sin \left(\frac{4 \pi}{\lambda}\left(x_{0}+\delta x_{s c}(t)\right)\right),
$$

where $L$ is the length of the cavity $(3 \mathrm{~km})$. The factor $K$ is defined as $\frac{\lambda}{4 L} \frac{T}{\sqrt{2 F \pi}}$ and the coupling factor $G$ as $K \cdot \sqrt{f_{s c}}$, which relates the motion of the scatterer (the bench) to strain noise. A similar model can be obtained for the other benches, but with $K$ depending on different optical parameters of the interferometer.

In practice the beam optical path undergoes slow drifts caused by thermal effects, air flows and beam alignment fluctuations. These drifts are of the order of a few microns in a time scale of some seconds. The angle $\phi_{0}$ can thus vary by more than $2 \pi$ within this timescale. The following considerations apply to Eq. 2:

- for small bench motion $\left(\delta x(t)<<\frac{\lambda}{4 \pi} \simeq 10^{-7} \mathrm{~m}\right)$ the equation linearizes as $h_{s c}(t)=G \cdot \cos \phi_{0} \cdot \frac{4 \pi}{\lambda} \cdot \delta x_{s c}(t)$. The term $\cos \phi_{0}$ accounts for the slow drifts, it 
averages to $1 / \sqrt{2}$ for time scales longer than one minute or so, while for shorter observation times the noise is non-stationary.

- for larger bench motion $\left(\delta x(t) \geq 10^{-7} \mathrm{~m}\right)$ the induced strain noise is nonlinear. This condition does occur in correspondence of mechanical resonances of the tables (bench and support structure, $f_{\text {res }} \simeq 10-20 \mathrm{~Hz}$ ), and at the microseism peak frequency $\left(f_{\text {res }}=0.3 \mathrm{~Hz}\right)$, which can be as large as some micrometers in case of intense sea activity.

One consequence of the non-linearity is that intense low frequency seismic excitations (below $10 \mathrm{~Hz}$ ) can produce noise in the detection band (above $10 \mathrm{~Hz}$ ). This up-conversion has indeed been observed in VSR2 and will be discussed in the following sections.

\subsection{Measurement}

Using the noise model of Eq. 2, the contribution of scattered light from the external benches can be estimated once the bench displacement and coupling factor are measured. One tri-axial seismometer (Episensor model ES-T) is placed on each optical bench, which measures the bench displacement in the frequency range from $0.2 \mathrm{~Hz}$ to $100 \mathrm{~Hz}$. Figure 1 illustrates the method of the coupling factor measurement. A mechanical shaker (Brüel \& Kjar model 4809) is used to seismically excite the bench with a sinusoidal signal. The signal amplitude is $A_{m} \simeq 10^{-6} \mathrm{~m}$ and the frequency is chosen close to the low frequency edge of the flat acceleration response function of the shaker $\left(f_{m} \simeq 20 \mathrm{~Hz}\right)$. Alternatively, a narrow-band white noise is used to excite the table mechanical resonance $\left(f_{m} \simeq 15 \mathrm{~Hz}\right)$.

The bench motion is measured by the seismic sensor (Figure 1(left)). The resulting noise $\left(h_{t o t}\right)$ in the interferometer is shown in Figure 1(right) compared to the quiet strain noise $\left(h_{0}\right)$ when no extra excitation is applied to the bench. The coupling factor $G$ of the model is adjusted to best reproduce the back-scattering noise $\left(h_{s c}^{2}=h_{t o t}^{2}-h_{0}^{2}\right)$.

Note the characteristic shape of the noise shown in Figure 1(right), which shows bumps at multiples of the frequency of the excitation signal. This is typically observed when, as in this case, the scatterer has essentially an oscillatory motion: $\delta x_{s c}(t) \simeq$ $A_{m} \sin \left(2 \pi f_{m} t\right)$. The back-scattering noise (Equation 2) is in this case a frequency modulated signal at $f_{m}$ with modulation index $m=A_{m} \frac{4 \pi}{\lambda}$. According to the JacobyAnger identity, the amplitude of the $n^{\text {th }}$ harmonics is given by the $n^{\text {th }}$ Bessel function. Harmonics are visible up to a maximum frequency $f_{\text {max }} \simeq m \cdot f_{m}$.

\subsection{Mitigation}

Noise produced by back-scattered light from the external benches was limiting Virgo's sensitivity at the end of VSR1 (Figure 2, left). The contributions of the individual benches are projected using the model with the coupling factor $G$ measured as explained 

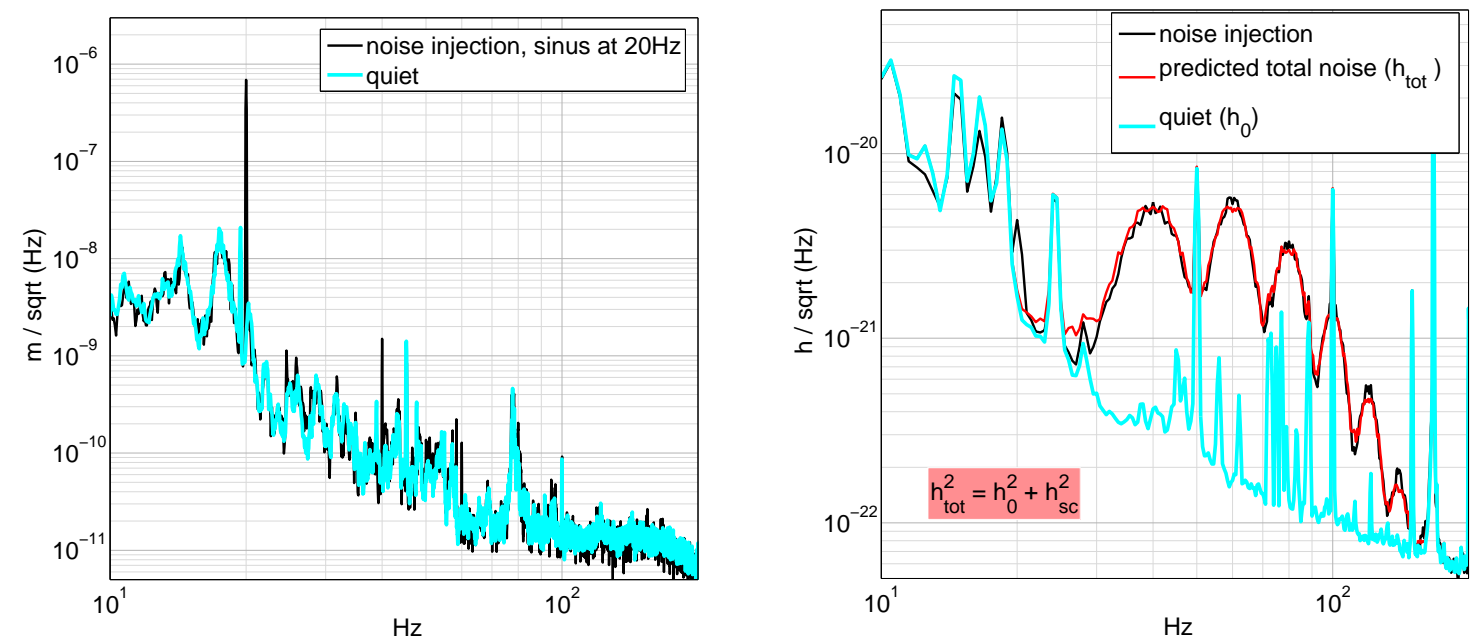

Figure 1. Measurement of scattered light coupling at the West-End bench. Pictures show the spectral composition of bench displacement (left) and interferometer output (right) in the undisturbed case (cyan) and when one sinusoidal excitation is applied to the bench (black). The excess noise in the interferometer output is matched closely by the scattered light model (red).

\begin{tabular}{|l||c|c|c|c|}
\hline measured $($ expected $)$ & $W E B$ & $N E B$ & $E D B$ & $E I B$ \\
\hline$G\left(\times 10^{-20}\right)$ before & 9 & 2.5 & 5 & 4 \\
$G\left(\times 10^{-20}\right)$ after & $2.3(2)$ & $0.15(0.5)$ & $1(1)$ & $<1$ (neglig.) \\
\hline
\end{tabular}

Table 1. Measured coupling factors of external benches, before and after the diffused light mitigation campaign. Values in parenthesis are derived from estimated $K$ and bench diffused light fractions.

in section 2.2, while using the displacement of the bench measured in quiet conditions. A lot of effort has been spent to mitigate this noise by acting in two ways [9]:

- reducing the motion of the benches at their resonance frequency (around $15 \mathrm{~Hz}$ ) by a factor 3: a resonant damper helped absorbing energy of the table mode, a new bench cover helped coupling less acoustic noise to the bench and a slowdown of the fans of the air-conditioning reduced seismic and acoustic noise emission.

- reducing the fraction of diffused light reflected by the benches.

Major sources of scattering on the bench were located by looking for bright spots with $I R$-cameras, as well as by tapping on optical elements and listening to the noise produced in the output signal of the interferometer. The amount of reflected light was reduced by misaligning lenses, dumping secondary beams, cleaning optics from dust particles and replacing standard mirrors by super-polished ones. For critical objects, the BRDF has been measured in a dedicated set-up, which allowed the estimation of the effective bench reflectivity $f_{s c}$ based on the optical geometry $[7,8]$. 

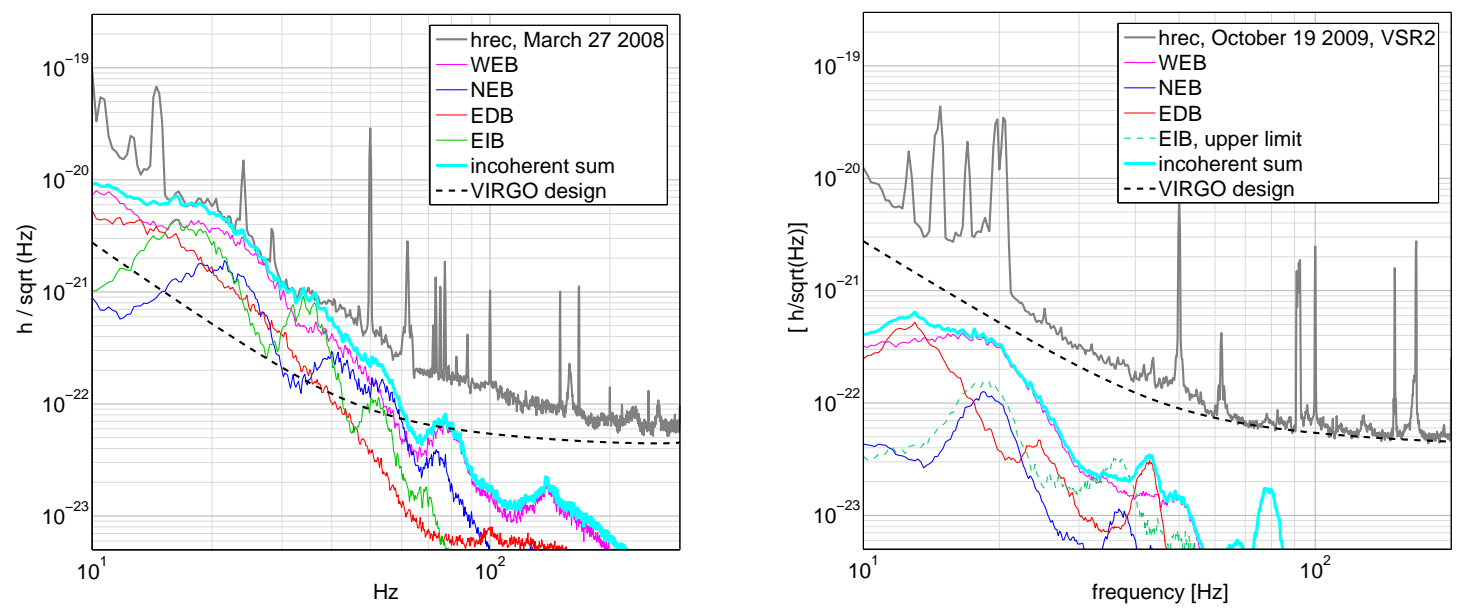

Figure 2. Projection of diffused light noise from external benches after VSR1 (left) and during VSR2 (right).

Table 1 shows the coupling factors for the various benches measured before and after the mitigation using the method of Sec.2.2. On average a reduction of a factor 2 to 5 was achieved. The measured coupling factors $G$ are in good agreement with values calculated using estimates for $K$ and $f_{s c}$. The large coupling of the West-End bench can be explained by the larger transmission of the West-End mirror ( $T=40 \mathrm{ppm})$ with respect to the North-End mirror $(T=10 \mathrm{ppm})$. Figure 2(right) shows the projection of benches scattered light noise during VSR2.

\section{Scattered-light noise effects in VSR2}

\subsection{Influence of bad weather}

During VSR2, it was observed that the largest changes in the sensitivity of the interferometer correlate with the presence of bad weather. The most significant effect of the bad weather is an increase in sea-activity, which typically follows strong winds with a delay of a few hours. This causes a large excess of seismic noise between 0.2 and $1 \mathrm{~Hz}$, see Fig. 3(left). Although the scattered light is not the only problem caused by bad weather, its effect on the output signal of the interferometer at low frequencies is the most obvious, see Fig. 3(right).

\subsection{Noise in the time-domain}

As can be seen in Eq. 2, the effect of a path-length change of the scattered light is periodic with a period of $\lambda / 2$. For fast path-length changes, the effect of the scattered light thus causes fringes in the interferometer output with frequency $f_{\text {fringe }}$

$$
f_{\text {fringe }}(t)=\left|2 \frac{v_{s c}(t)}{\lambda}\right|,
$$



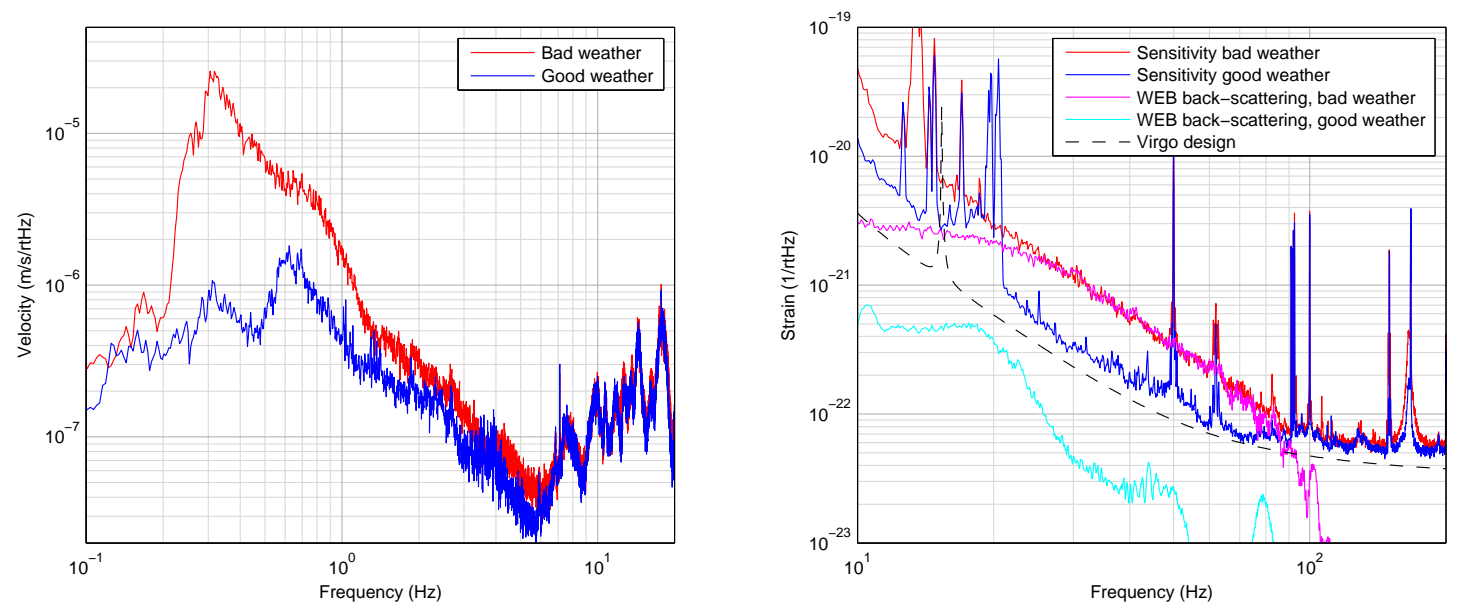

Figure 3. Comparison between good and bad weather. (left) Linear spectral density of the velocity of the West-End Bench along the beam axis, reconstructed using position sensors and accelerometers. The big increase below $1 \mathrm{~Hz}$ is caused by sea-activity, while the structure around $10 \mathrm{~Hz}$ is caused by bench resonances. (right) Measured sensitivity of the interferometer and projection of scattered-light noise. In bad weather conditions, this noise is limiting up to $100 \mathrm{~Hz}$.

with $v_{s c}$ the velocity of the scatterer (the derivative of $\delta x_{s c}$ ). This velocity can be measured by various sensors, since at the low frequency of the microseism, the earth and the optical bench move synchronously, while the mirror is kept fixed by the seismic isolation system and the control system of the interferometer. Best results were obtained by taking the derivative of a position sensor of the seismic isolation system.

The low frequency oscillations caused by the microseism produce pretty typical arches in the spectrogram of the output of the interferometer, see Fig. 4(left). The frequency as a function of time is almost perfectly predicted by the model of Eq. 3 for the velocity of the West-End bench, whose noise contribution is dominating.

Also the second harmonic frequency of the arches can be observed in the spectrograms, see Fig. 4(left). This can be explained by scattered light that follows a double-bounce path, in which the light is transmitted by the end-mirror, reflects off some moving component on the bench, reflects off the high reflective end-mirror and scatters a second time on the bench before reentering the main beam. In this case, the optical path of the scattered light changes with 4 times the change in position of the ground, instead of 2 times as seen in Eq. 3. In this case, the induced contribution to the output of the interferometer is thus

$$
h(t)=G_{2} \cdot \sin \left(\frac{8 \pi}{\lambda} \cdot x(t)\right)
$$

Since this involves two spurious reflections, it would be expected that $G_{2}$ is many orders of magnitude lower than $G$. In reality it was found that $G_{2} \simeq 6 \times 10^{-21}$ at the WestEnd bench, only a factor 4 lower than $G$. One possibility is that one of the scatterers has a very high reflection (e.g. a photo-diode or an uncoated glass surface). Most 

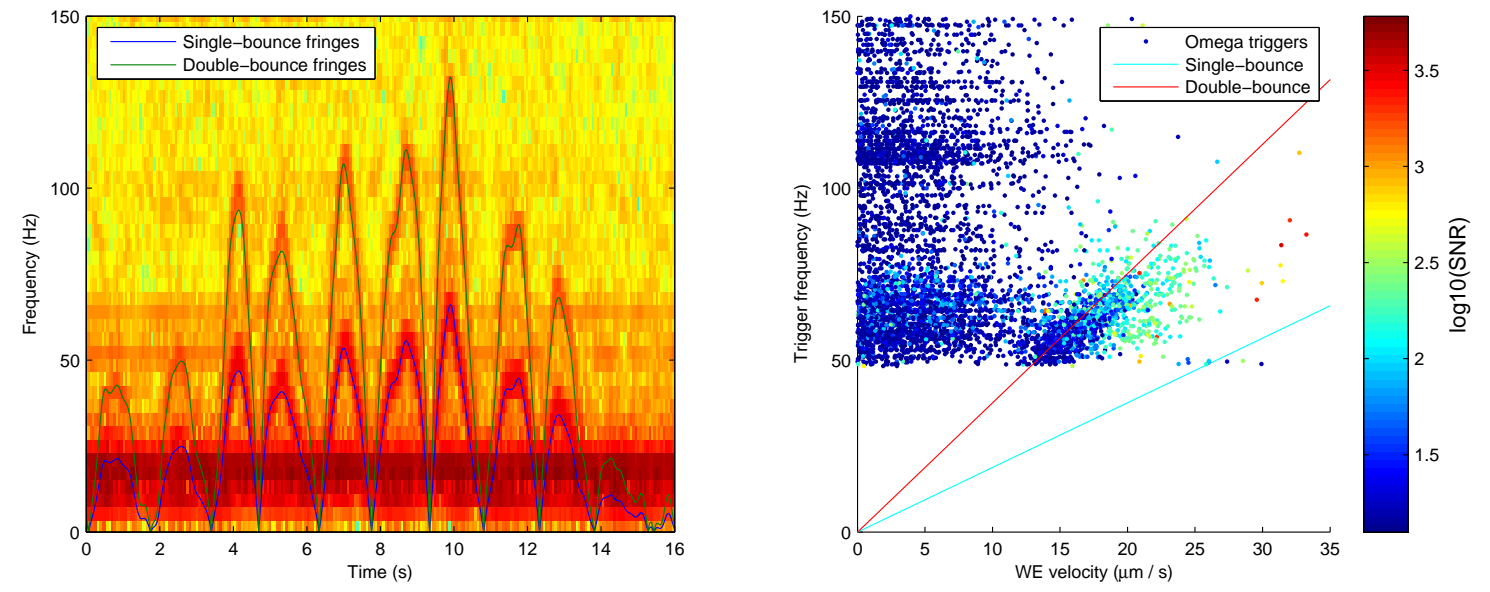

Figure 4. (left) Spectrogram of the interferometer typical output signal in conditions of intense microseism activity, the color scale is logarithmic. Overlaid are curves calculated with Eq. 3 and Eq. 5 using the measured speed of the West-End Bench, without any adjustment of parameters. (right) Scatter-plot of glitch frequency vs ground velocity at the moment of a glitch. This plot displays the glitches collected by the Omega pipeline during one day with high microseism. The SNR of the glitch is indicated by the color scale. The colored lines indicate the relations of Eq. 3 and Eq. 5.

of these components are tilted to not scatter directly back into the beam, so there is probably some unfortunate geometrical path that allows such a reflection to reenter after a reflecting once off the end-mirror.

One problem with the double-bounce fringes is that they occur at twice the frequency of the normal fringes:

$$
f_{\text {fringe } 2}(t)=2 f_{\text {fringe }}=\left|4 \frac{v_{s c}(t)}{\lambda}\right|
$$

Even though $G_{2}$ is lower, these fringes have a larger impact on the sensitivity of the interferometer since the noise occurs at higher frequencies, where Virgo is more sensitive.

\subsection{Transient events}

One type of gravitational-wave signals that is sought in the output of the interferometer is short and burst-like. The data coming out of the interferometer is analyzed by various software pipelines to find these signals. Since the disturbances caused by scattered light can also be short and burst-like (the arches in the spectrogram described before), they are detected by the pipelines and cause a large amount of false triggers in case of bad weather.

One of these pipelines, called Omega, is looking for sine-Gaussian type bursts in the whitened output signal of the interferometer [10]. For each trigger, it records the time, frequency and signal-to-noise-ratio (SNR). As indicated by Eq. 3 and Eq. 5, the fringe frequency depends on the velocity of the scatterer. To test if this relation also holds 
for the observed glitches, the reported frequency has been plotted against the velocity of the West-End bench, interpolated at the moment of the glitch, see Fig. 4(right). Indeed, a distinct cloud of high-SNR points is clearly visible, which are well predicted by the model for the double-bounce glitches. Note that the pipeline does not report at frequencies below $50 \mathrm{~Hz}$, where the interferometer is less sensitive. A procedure to veto these glitches based on the measured velocity is being studied [11].

\section{Conclusions}

Light back-scattered by in-air optics can be a critical source of noise to gravitationalwave interferometers, since the phase of the reflected light is modulated by the motion of the optical benches, which is driven by natural and anthropogenic seismic noise. In case of large motion of the optics, the low-frequency seismic noise can up-convert into the detection band of the interferometer and severely reduce the sensitivity of the instrument. This phenomena has been thoroughly studied in the last years and its effect on the sensitivity is now accurately modeled. The noise can be prevented by reducing either the amount of back-scattered light or the motion of the scatterer.

Procedures have been developed to measure the coupling factor of this noise to the gravitational-wave signal. This was essential to identify the problem and evaluate improvements. Due to a large effort to reduce the amount of scattered light and the environmental noise on the optical benches, the noise produced by scattered light is now not limiting Virgo's sensitivity in quiet conditions. It is, however, still an issue in periods with high microseism. This was evident during Virgo's second science run, which showed a large increase in the glitch rate in case of bad weather. This problem might be partly mitigated in the data analysis by implementing a veto based on the ground velocity.

Using the obtained experience, actions are planned to suppress the scattered-light noise to well below the design sensitivity of future upgrades (Virgo + and Advanced Virgo). Direct improvements are expected from the increase of the finesse of the FabryPerot cavities, a reduction of the transmission of the end-mirrors and the addition of antireflection coating that was missing on some output windows. It will remain necessary to prevent as much as possible the reflection of light from the external benches. This will be done by careful design of the optical layout of the benches, a choice of low-scattering optical components and the efficient dumping of spurious reflections. For Advanced Virgo, the main photo-diodes will be located under vacuum on seismically isolated benches. An active seismic isolation system is considered for the external benches.

\section{References}

[1] Accadia T et al. 2010 Commissioning status of the Virgo interferometer Class. Quantum Grav. 27 084002

[2] Vinet J-Y, Brisson V and Braccini S 1996 Scattered light noise in gravitational wave interferometric detectors: Coherent effects, PRD 54,2 1276 
[3] Vinet J-Y, et al 1997 PRD 56,10 6085 Scattered light noise in gravitational wave interferometric detectors: A statistical approach.

[4] Takahashi R, Arai K, Kawamura S and Smith M R 2004 Direct measurement of the scattered light effect on the sensitivity in TAMA300, Phys. Rev. D 70, 062003

[5] Lück H et al 2008, Opto-mechanical frequency shifting of scattered light, J. Opt. A: Pure Appl. Opt. 10085004

[6] Acernese F et al. 2008 Class. Quantum Grav. 184003 Noise studies during the first Virgo science run and after

[7] Canuel B, Determination of back-scattering from single optics, Virgo note in preparation.

[8] Tournefier E 2008 Back-scattering by the optical benches: results from Virgo and constraints for AdV. Virgo Internal Note VIR-NOT-070A-08 (available at https://tds.ego-gw.it/ql/?c=2083)

[9] Canuel B, Fiori I, Marque J, Tournefier E 2009 Diffused light mitigation in Virgo and constraints for Virgo+ and AdV. Virgo Internal note VIR-0792A-09 (available at https://tds.ego$\mathrm{gw} . \mathrm{it} / \mathrm{ql} / ? \mathrm{c}=7118)$

[10] Chatterji S et al. 2004 Multiresolution techniques for the detection of gravitational-wave bursts. Class. Quantum Grav. 21 S1809

[11] Robinet F for the LIGO Scientific Collaboration and the Virgo Collaboration 2010, Data Quality in Gravitational Wave Burst and Inspiral Searches in the Second Virgo Science Run. Submitted to CQG (should appear in the same issue) 